\title{
Anomalies identification in PV isolated system
}

\author{
A. Lopez-Aguera ${ }^{\mathrm{a}}$,I. Rodriguez-Cabo ${ }^{\mathrm{a}}$, C.M. Torres Costa ${ }^{\mathrm{b}}$ \\ a Department of Particle Physics \& Galician Institute of High Energy Physics \\ Astroparticle Group. Santiago de Compostela University \\ ${ }^{\mathrm{b}}$ Department of Chemical Engineering \& Aula de Enerxias Renovables \\ South Campus 15702 Santiago de Compostela (Spain) \\ phone:+34 981 563100, fax:+34 981521091, e-mail: lopez@fpaxp1.usc.es, \\ ircabo@fpaxp1.usc.es,carmenmari.torres@rai.usc.es
}

\begin{abstract}
A quality check procedure for early detection of photovoltaic (PV) isolated system anomalies is presented. The proposed method is developed over a short set of monitored system parameters. The cause of the anomaly is also identified and a software alarm is sent to the controller. The method as been developed over a sample of $10^{5}$ experimental data collected in the grid of 300 PV from the Pierre Auger Project.
\end{abstract}

\section{Key words}

Solar energy, PV system's online diagnosis, system failures, stability, batteries.

\section{Introduction}

Most of the stand-alone PV systems applications are characterized by a continuously energy requirement. In order to prevent a lack of energy supply, a quality check procedure for early detection of PV isolated system anomalies is needed.

The average operating lifetime of the batteries is lower than in the case of the other components of the system. They have to be replaced typically between 4 and 5 times during the expected lifetime of the whole system. Therefore batteries has an important role in isolated PV systems' performance, and anomalies in their shape are common causes of system troubles. In the following, a quality test for early battery anomalies detection is described, using the battery voltage distribution as a tool with which find the problem before the lack of energy supply occurs.

This is the case of the power supply of the ground detectors at the Pierre Auger Observatory[1], which consist in a macro-grid of isolated PV systems forming an hexagonal network composed of 1600 particle detector stations. Each $100 \mathrm{Wp}$ station includes $253 \mathrm{~W}$ panels, a MPW (Pulse-with modulation) regulator which ensure a stable charge-discharge process and 2 seried 105 Ah lead-acid batteries.

The experiment has an expected lifetime of 20 years, during this period main PV system values are continually monitored each 10 minutes. The data analysis of this experimental set-up constitute an important database of about $10^{5}$ data in the first 2 years of normal operation, so it represents a excellent opportunity for the study of stand-alone PV systems apart from its own scientific aims.

\section{Method for Anomalies identification}

The analysis of the battery voltage distribution, $f(V)$, is proposed as an indicator of the state of health for the PV system in general, and for the battery in particular. In the $\mathrm{f}(\mathrm{V})$ distribution we define four operational regions:

1) Deep discharge region where the voltage is lower than $11.8 \mathrm{~V}$ in the battery;

2) Discharge region (voltage between $12 \mathrm{~V}$ and 13 $\mathrm{V})$;

3) Regulation region where the battery voltage is in the interval $[14,15] \mathrm{V}$;

4) Over charge region where the voltage is higher $15.5 \mathrm{~V}$ in the battery.

The frequency in which each battery is working in each region can be used as an indicator of anomalies legated to different periods in the battery performance. Three criteria are used in the battery anomaly identification based on the distribution percentage for each region respect the total frequency: \% in deep discharge, $\%$ in regulation and $\%$ in overcharge.

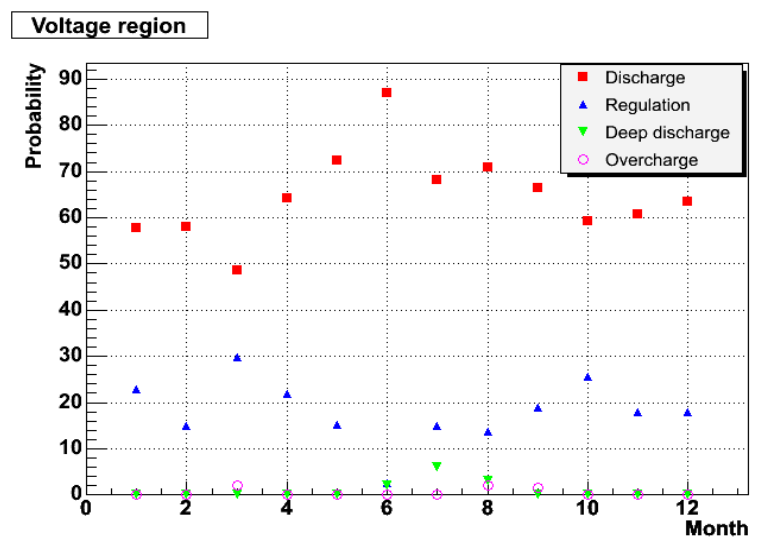

Figure 1. Probability along the year for each working region. A sample of well working system has been used. 
Figure 1 shows this probability along the year for each working region. Deep discharge and over voltage probabilities constitute a few percentage in normal working conditions. Dependence of $f(V)$ with both irradiation and temperature need to be taken into account. Therefore, as it can be seen, the deep discharge increases in the months of winter, June, July and August in this case, while the regulation decreases.

\section{A. Deep discharge}

The voltage probabilities in deep discharge should constitute a few percent less than $0.1 \%$ in normal working conditions, for this reason, those batteries with an extremely high integral of the frequencies corresponding to the deep discharge region has an anormal performance which can be an indicator of an anomaly. Figure 2 shows the deep discharge probability for 300 PAO stations.

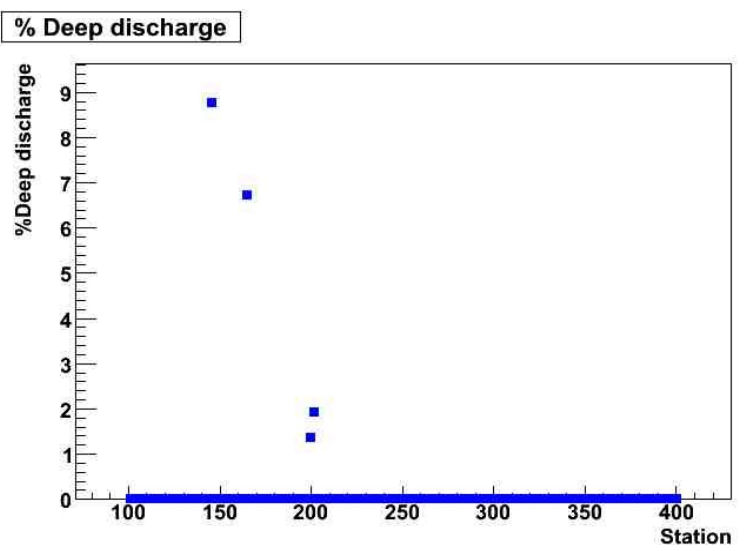

Figure 2. Probability of deep discharge for each arbitrary numerated station in March 2005.

In this cases the battery is quickly discharged because of his reduced capacity, or there is a problem with the charge, which means that it is not been charged correctly due to a bad connection or a regulator fail. The effect is a serious problem which produces a strong sulfation that could be irreversible.

\section{B. Regulation}

As in the case before, the percentage of time in which the battery is in the regulation region could be analyzed to find an anormal performance.

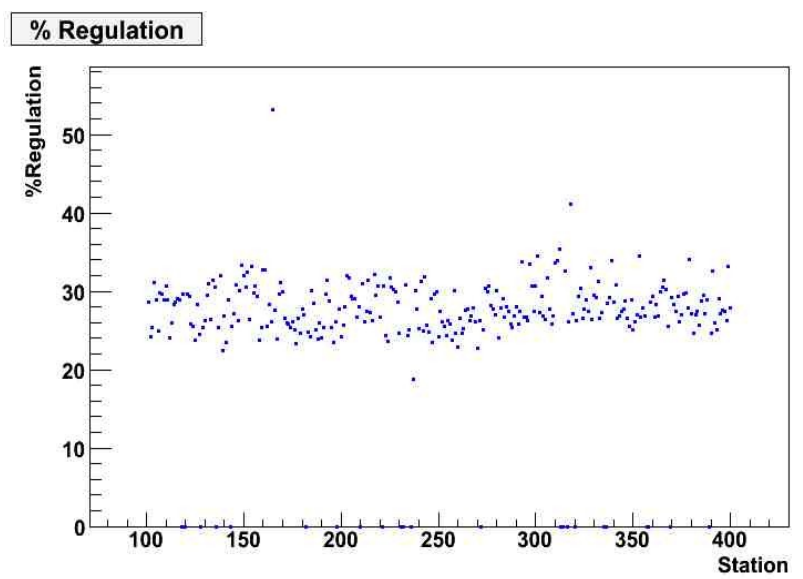

Figure 3. Frequency percentage corresponding to the regulation region for each numerated station in March 2005.
Figure 3 shows, as example, the behaviour of 300 stations for a particular month. The distribution of the \% of regulation corresponding to an enough number of stations, fitting to a Gauss function (see figure 4), allows us to obtain parameters with which fix the normal limit values. A percentage of regulation below the low normal limit can be cause by either connection failures or problems with the regulator. On the other hand, a regulation percent higher than the normal limits is normally related to a bad performance of the regulator. Both cases need to be careful controlled.

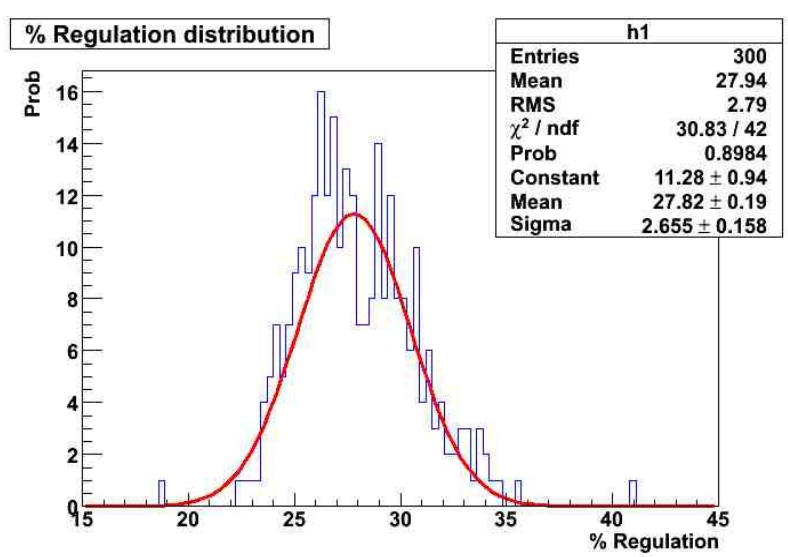

Figure 4. Distribution of the regulation percentage from 300 stations in March 2005, fitting to Gauss function.

\section{Overcharge}

A battery supporting voltages over $15.5 \mathrm{~V}$ for long periods of time gasifies, loosing liquid and causing corrosion problems. The effect is a fast decrease on the battery capacity and then on the lifetime. The regulator plays a key role, in fact, this type of problems are legated to its operation. One reason may be that one of the seried connected battery in a station is in deep discharge and the regulator, which acts according to the total voltage of both batteries, unbalances the other. On the other hand, an over voltage can be produced when the regulator has an excessive frequency of pulses while the regulation time.

As in the previous case, the distribution percentage corresponding to the overcharge region can be used to find a gasified candidate battery.

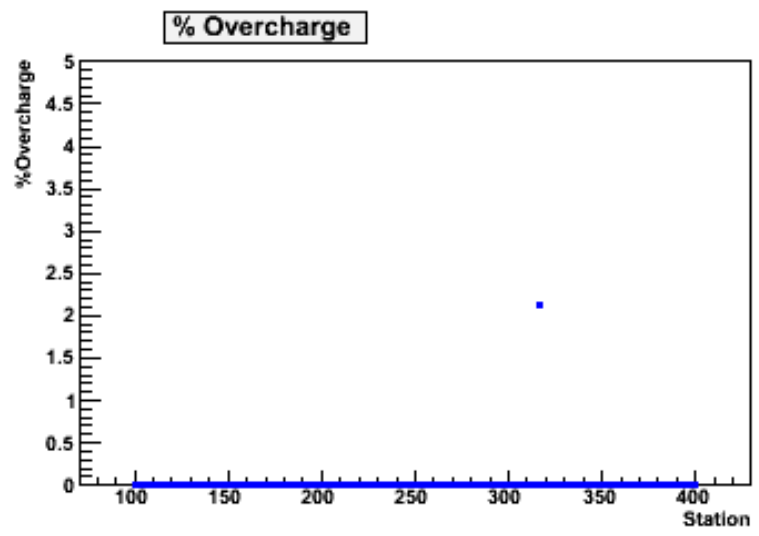

Figure 5. Probability of overcharge for each arbitrary numerated station in March 2005. 
As figure 5 shows, a few number of batteries present a significant overcharge percentage, in this case only 1 of 300. This problem lead to a fast decrease on the battery capacity and then on its lifetime. In general, for a good performance battery, the expected percentage is lower than $1 \%$, even in summer.

\section{Systematization procedure}

In order to analyse the validation of the quality test previously proposed, a computerized program has been created using the tool software ROOT[2]. As the input the program use the monitored data of voltage of each station. To avoid seasonal dependences, the time sensibility is fixed to 1 month. As the output we produce a file in which are registered all the stations that are candidate to be in bad performance. It also includes a code of possible causes of the anomaly.

In a first stage, the computerized program calculates the percentage values that correspond to each voltage region for a sample of $300 \mathrm{PAO}$ stations. And this operation is repeated for each month.

For the identification of the regulation anomalies a histogram with all the regulation percentages of the batteries is created with a monthly frequency. Fitting these distributions to a Gauss function we obtain the corresponding parameters, mean and RMS, with which we can fixed limits. A battery with a percentage out of this range is candidate to suffer an anomaly. The range is fixed using a standard 2 sigma criteria.

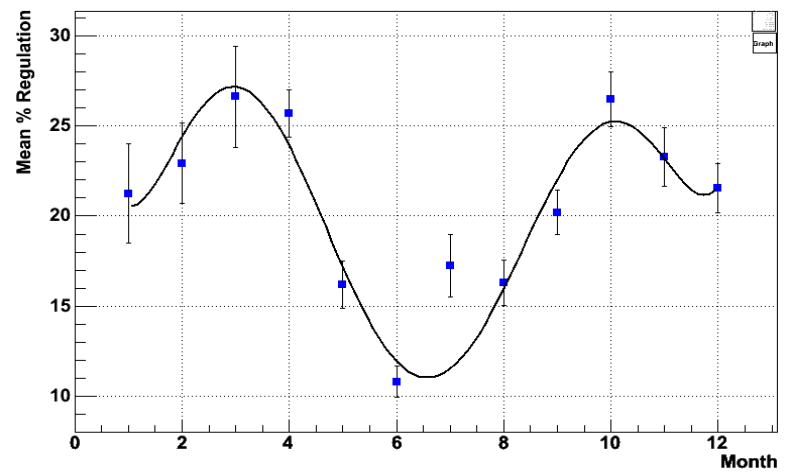

Figure 6. Variation on the mean regulation percentage during

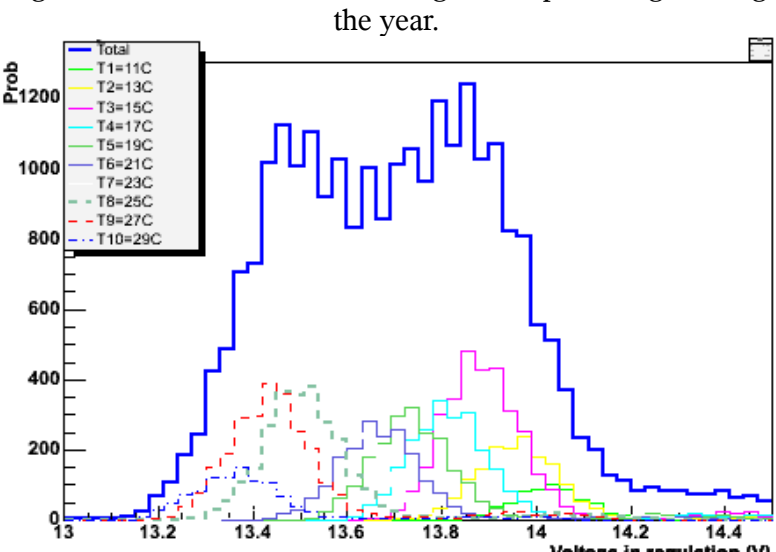

Figure 7. Voltage distribution in regulation regime for a standard working station. In blue the global distribution. Each of the small graphics included shows the distribution for a discrete temperature interval.
Figure 6 shows the variation in the mean regulation percent during the year 2005 for a sample of 300 stations. Variations are directly related to the weather conditions. Another view of the same effect is shown in figure 7.

Perform a quality check procedure, implies send software alarms which alerts of the anomalous situation of the PV system performance in a systematic way. The systematization implies define constant cutting parameters, it means independent of weather conditions. In particular, temperature and irradiation level. The experimental set-up location, in a extra-clean atmosphere, simplify the normalization because, in first approximation both variables are linearly correlated. Let study the battery's voltage dependence with the temperature. Figure 8 shows the linear dependence. A linear parametrization is straight forward.

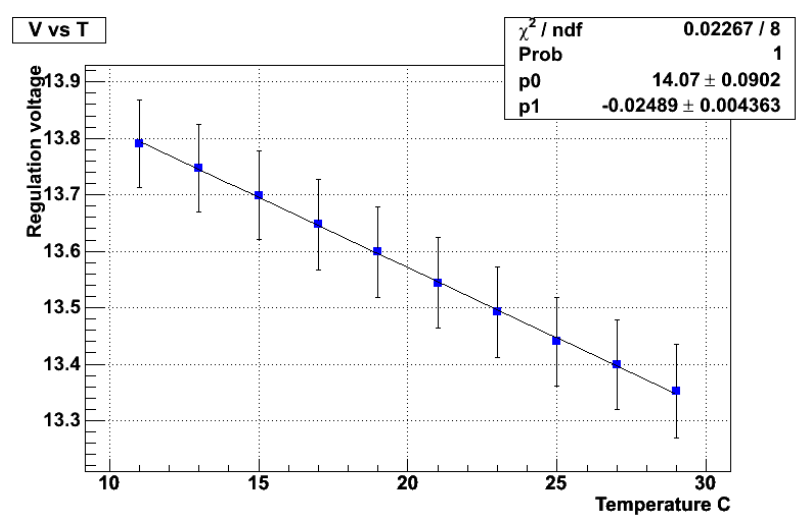

Figure 8. Voltage regulation dependence with the temperature.

Once corrected, if the probability in the deep discharge region associated to a particular station is higher than $0.1 \%$, the program will identify an anomaly for that certain month. The frequency of the presence of anomalies is evaluated and used as parameter for further actuations.

In order to detect a battery which works in the overcharge region a similar procedure is carried out. In this case those stations with a percentage in the overcharge region higher than a $1 \%$ are identified.

As final output, the program produced a file with the alarms registered. This file includes the numbers of the stations, the month in which the alarm has been detected and a code for each anomalous case. The resulting analysis is graphically represented and it is showed in figure 9.

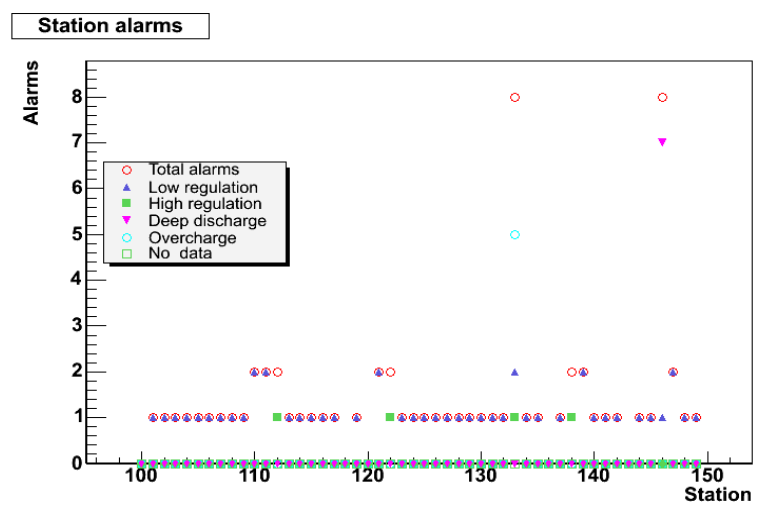

Figure 9. Number of alarms for 50 PV stations. 
As it can be seen in figure 9, the most common anomaly detected is in the regulation regimen, but it only appears in one or at least two occasions (months) during the year so it could be legated to a problematic period of the year with a low supply of energy to the batteries.

Apart from that, it is clear that stations 133 and 147 have to be studied in detail. Station 133 has a problem of overcharge because it gives this type of alarm in five occasions, so it is a gasified candidate battery. In the case of station 147 the alarm of a deep discharge anomaly appears 7 times during the year, then it can cause a strong sulfation problem in the battery.

\section{Identification of anomalies causes}

With anything else but the study of the normal working performance of a PV system, and using the information obtained in the log-book, it is possible to identify the main effect of the anomaly as well as the possible causes. This identification, allows to add to the alarms information, a probable cause in order to make easier the work of the maintenance team. In figure 10, we show the scheme of both detection procedure (red squares) and identification causes suggested (blue squares) as well as de effect produced.

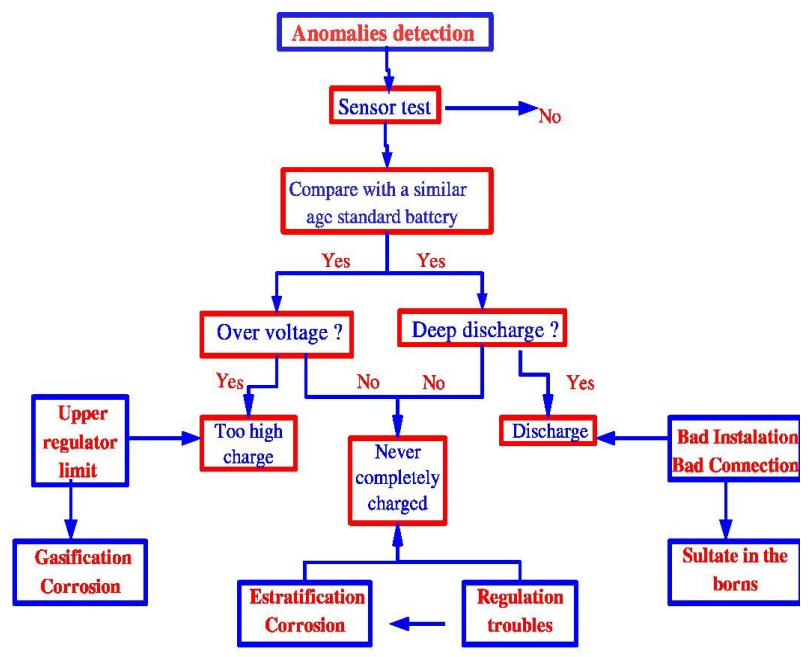

Figure 10. Scheme for PV isolated system anomalies detection and identification (red squares). Main causes and damage effect is marked inside blues squares.

\section{Example of a non early anomaly detection}

As an example of a battery that has presented an anomaly and that with this procedure could have been detected is the battery 1 of the station number 243, that was installed in October 2003. Each element of the system was checked before its installation in the field. Although, from the beginning during the normal operation battery 1 was never completely charged.

Figure 11 shows the history of a battery damaged. In November of 2003 shows an anomalous behaviour reaching considerably low values of voltage. In the following months, the battery goes on showing the same performance and degenerating into working conditions so obviously it became damaged, and therefore it has to be replaced between August and September of 2004.
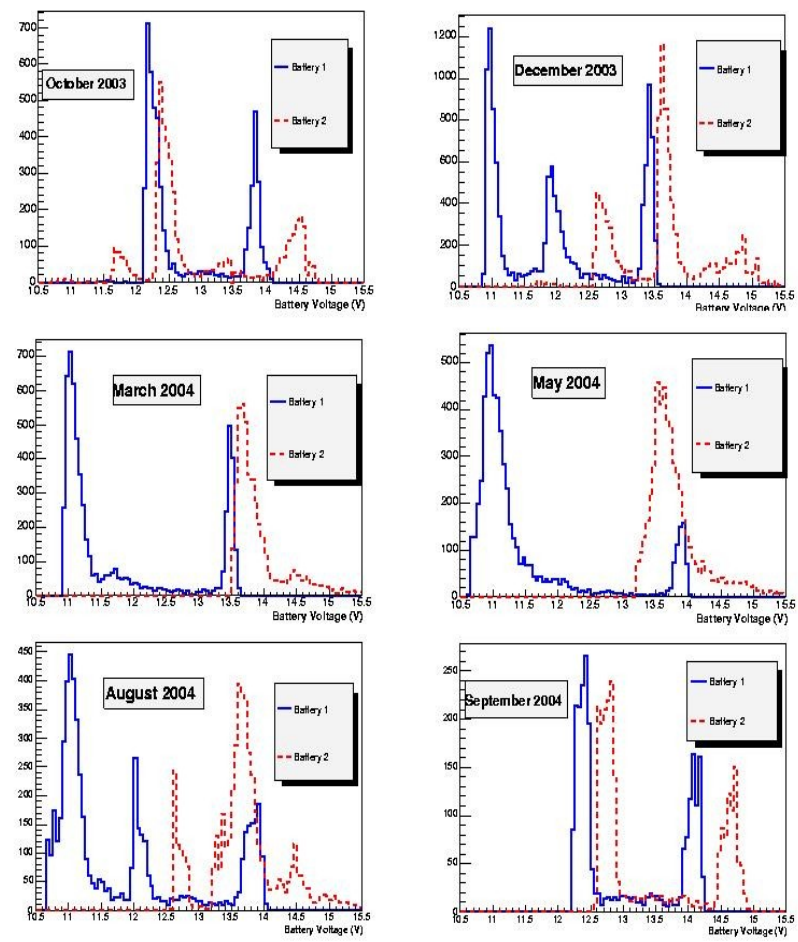

Figure 11. Evolution in the voltage distributions for the two batteries of the station 243 .

Moreover, due to in our PV system both batteries are seried, the safety cuts on the regulator affect the sum of both batteries voltages. For this reason the damage affect both batteries at the same time: One suffer a frequent deep discharge while the other battery is in over voltage. Due to this situation the battery 2 stays in high voltage values so it can gasifies and produce corrosion. The deviation of about $0.6 \mathrm{~V}$ observed between batteries is artificial.

Unfortunately the battery 1 was neutralised. The battery 2 presented loss of liquid. We recovered the system with the installation of a new battery 1 . Battery 2 was recharged and tested. The battery was cataloged as no good and re-used.

\section{Conclusions}

The expected lifetime of a PV system is at least twenty years of continuos work. The only way to prevent system failures is an early detection of possible problems. Main common troubles appears legated to the solar panel connections, the regulator shape as well as any anomaly in the batteries shape. The proposed quality check for early anomalies detection is based on the battery voltage distribution and the ambiental temperature.

The results obtained over a sample of $300 \mathrm{PV}$ of the PAO show how less than $1 \%$ of the batteries suffer from deep discharge, while $0.3 \%$ work on overcharge regime during 1 year of operation.

The effect of a large delay in failures detection is experimentally described.

Once demonstrated the utility of the quality check method, the procedure will be implemented as standard on-line check in the data acquisition system of the PAO. 


\section{References}

[1] "The Pierre Auger Project". Nucl. Instrum. Meth. A523 5095, 2004. General information about the project is available at www.auger.org.ar

[2] ROOT www.cern.root.ch

[2] D. Linden, Handbook of Batteries, McGrawHill, New York (1995).

[3] A. Jossen, J. Garche and D.U. Sauer, Operation conditions of batteries in PV applications, Solar Energy, 76, 759769, (2004).

[4] I. Rodriguez Cabo. Graduation Thesis. USC. 